\title{
A la zaga de una propuesta para la combinación de metodologías de investigación
}

Chasing the proposal for the combination of research methodologies

\author{
Maria Victoria Rugeles Gélvez \\ Magistra en Comunicación, Universidad Santo Tomás \\ mrugelesgelvez@yahoo.com \\ Eliana del Rosario Herrera Huérfano \\ Magistra en Comunicación, Corporación Universitaria Minuto de Dios \\ rhhuerfano@yahoo.com \\ Carlos Andrés Muñoz Sandoval \\ Sociólogo, Universidad Santo Tomás \\ camsfun_@hotmail.com \\ Artículo de investigación
}

Fecha de recepción: 8 octubre de 2012 • Fecha de aprobación: 18 de noviembre de 2012

\section{RESUMEN}

Fuera de sus resultados, pocas veces las investigaciones exponen el proceso creativo de definir y poner en marcha una estrategia metodológica. Este artículo presenta una descripción y reflexión sobre la propuesta metodológica diseñada para el proyecto "Experiencias de comunicación y desarrollo sobre medio ambiente en la región andina de Colombia”. Inicialmente se detalla el contexto del proyecto, seguido por las referencias conceptuales, la descripción del diseño metodológico y los procesos de recolección y sistematización en cada fase, con sus respectivas dificultades, a partir de lo cual se derivan aprendizajes que ocupan la parte conclusiva del artículo.

Palabras clave: investigación social, metodologías, comunicación, desarrollo, medio ambiente. 


\begin{abstract}
Research tends to show results and only in some specific instances, it exposes the creative process and the implementation of a methodological strategy. This article presents a description and an analysis of the methodological strategy designed for the study "Experiences on communication and development of the environment in Colombia's Andean region". Initially it explains the context of this project, then it highlights conceptual references, it describes the methodological design and the processes of gathering and classification of information in each phase, it mentions the difficulties, and the lessons derived from this project are also discussed at the end of this article.
\end{abstract}

Keywords: social research, methodologies, communication, development, environment.

\title{
CONTEXTO DEL PROYECTO
}

Este documento se originó a partir del proceso de investigación "Experiencias de comunicación y desarrollo sobre medio ambiente en la región andina de Colombia", apoyado institucionalmente por la Universidad Santo Tomás, la Corporación Universitaria Minuto de Dios y la Universidad Nacional Abierta y a Distancia. Los autores agradecen y reconocen la participación, mediante discusiones, del resto del equipo, integrado por Ana María Guerrero Martínez, María Isabel Noreña Wiswell, Andrea Sotelo Carreño, Patricia López Preciado y Germán Sáenz; así como los aportes, en su momento, de Álvaro Herrera Arango y César Rodríguez Charry.

La mencionada investigación continúa un trabajo iniciado en el 2006, cuando se llevó a cabo un primer proyecto denominado "Estado del arte de la investigación en comunicación y desarrollo". Este trabajo compendió los estudios realizados y recapituló la forma como se ha abordado, desde la academia, la relación comunicación-desarrollo. El segundo proyecto, del cual surge esta reflexión, directamente confrontó la relación con trabajos específicos gestionados por organizaciones sociales, medios de comunicación e instituciones públicas y privadas que buscan la transformación social de problemáticas medioambientales en una región de nuestra geografía nacional: la región andina. 
De esta forma, surgió la investigación "Experiencias de comunicación y desarrollo sobre medio ambiente en la región andina de Colombia". En las discusiones preliminares del diseño se tenía clara la idea de comprender la relación comunicacióndesarrollo desde experiencias cotidianas. La propuesta se originó en una presentación del proyecto "Estado del arte de la investigación en comunicación y desarrollo", en el marco de la Cátedra Unesco sobre Comunicación, Desarrollo y Cambio Social, organizada en 2007 por la Pontificia Universidad Javeriana. Una vez concluida la presentación, un activista de la comunicación, bastante comprometido con la generación de transformaciones sociales a través de procesos comunicativos, interpeló a los investigadores sobre la búsqueda del sentido de esta relación en la interpretación que hacen los académicos desde las universidades, y no desde la experiencia de quienes hacen la comunicación. Desde ese momento, la propuesta fue acogida.

Dado que el universo de experiencias en comunicación y desarrollo puede resultar desbordante, los investigadores decidieron tratar de acotar los alcances del proyecto y revisar experiencias que se autorreconocieran como tal (es decir, en el marco de propuestas de comunicación y desarrollo) y estuvieran enfocadas en una temática en particular: el medio ambiente.

Para establecer la delimitación desde el tema, se contemplaron los resultados arrojados por el estado del arte, a partir de lo cual se evidenció que tres de las treinta y nueve investigaciones analizadas trabajaban sobre lo medioambiental como eje central. Junto al problema del medio ambiente se pensaron otras posibilidades de temas que contribuyeran a acotar el objeto de estudio: inclusión y exclusión étnica y desplazamiento. Finalmente, el grupo decidió trabajar con experiencias de comunicación y desarrollo sobre medio ambiente, por las implicaciones futuras que la situación ambiental impone a la humanidad.

Una última definición se centró en pensar el cuerpo de la investigación: se contempló que abarcar todo el territorio colombiano podría ser bastante ambicioso, por lo que se determinó trabajar en la región andina de Colombia, en consideración con que es la más densamente poblada: según el censo del Departamento Nacional de Estadística (2005), en ella se ubica el 74 \% de la población colombiana; por esta razón, también es la más activa económicamente. La región andina ocupa una gran extensión del 
territorio nacional: va desde el suroccidente del país, donde la cordillera de los Andes se divide en otras tres cadenas montañosas, hasta el nororiente colombiano. $\mathrm{Su}$ relieve está conformado por valles, nevados, mesetas, páramos, volcanes y una gran riqueza hidrográfica, lo que en conjunto representa un privilegio para el país, por la diversidad de recursos naturales.

Esa diversidad se encuentra amenazada por el uso irresponsable de los recursos, bien sea desde la cotidianidad de la sociedad en general o desde los macroproyectos avalados por el gobierno local, regional o nacional, con una visión de la producción en términos meramente económicos y sobre los cuales no se publica información clara en los medios de comunicación. En contrapeso, se encuentran también múltiples experiencias de comunicación y desarrollo que surgen en respuesta a ese uso irreflexivo de los recursos naturales, cuyos objetivos van desde crear una conciencia ecológica colectiva, hasta generar proyectos educativos e informativos sobre problemáticas medioambientales concretas, como el manejo de basuras, la contaminación, el manejo de agroquímicos y la deforestación.

En este contexto, la investigación pretendió mapear, aprehender y dar a conocer estas experiencias de comunicación, centrando la comprensión de la relación comunicación-desarrollo sobre medio ambiente en diez estudios de caso.

\section{REFERENCIAS CONCEPTUALES DE PARTIDA}

Inicialmente fue preciso un ejercicio de grupo de estudio para abordar la reflexión y discusión conceptual sobre desarrollo, comunicación y medio ambiente, con el cual se llegó al reconocimiento, desde diferentes miradas, de las relaciones entre comunicación-desarrollo, desarrollo-medio ambiente y comunicación-medio ambiente, para lograr construir una propuesta de visiones sobre la relación comunicación-desarrollo-medio ambiente.

Desde la perspectiva de la relación comunicación-desarrollo, se partió de la propuesta de Gumucio-Dragon (2004) y Waisbord (2001) sobre las tendencias que explican esta relación: 1) el paradigma dominante sustentado en la visión de la teoría de la modernización, en el cual la relación se construye desde el marketing social, la 
promoción de la educación y la salud, y el eduentretenimiento; 2) las críticas al paradigma dominante, en las que se destaca la teoría de la dependencia, desde la cual se dio lugar a las experiencias de comunicación para el desarrollo, de corte más institucional, y a las experiencias de comunicación alternativa desde los excluidos; 3) en la última etapa histórica de estas críticas al paradigma de dominante, se ha potenciado la perspectiva de la participación y la movilización social en el marco de lo que hoy se reconoce como comunicación para el cambio social. Estas tendencias permitieron proponer, desde el estado del arte, tres categorías para abordar la relación: comunicación para el desarrollo, comunicación alternativa y comunicación para el cambio social (Herrera y Uruburu, 2009: 120).

La puerta de entrada en la reflexión sobre el tema ambiental fue la ubicación histórica en la segunda posguerra, cuando la preocupación por los recursos naturales se agudiza con la crisis del petróleo y el marcado interés que paulatinamente va surgiendo sobre el control (principalmente económico) frente a la apropiación, explotación y manejo de recursos no renovables en el mundo.

Para el proceso de discusión, el equipo consideró diferentes fuentes: los aportes conceptuales sobre valor utilitario (económico) y valor no utilitario (sociocultural, intrínseco y ecológico) que permiten develar diferentes relaciones entre medioambiente y desarrollo (Penna y Cristeche, 2008); las reflexiones sobre la espectacularización y fragmentación en el tratamiento informativo del tema medioambiental (Cimadevilla, 2005); el cuestionamiento sobre la ausencia del tema en la esfera de lo público (Seoanez, 1997); la propuesta de relaciones entre economía ecológica, ecología política y conciencia ecológica (Leff, 2003), y las reflexiones sobre comunicación y participación en el manejo de los recursos naturales, promovidas desde los documentos de la Organización de Naciones Unidas para la Alimentación y la Agricultura (FAO) $(2003,2004,2008)$.

En esta medida se reconocen, desde la propuesta de Michael Colby (citado en Penna y Cristeche, 2008: 18), cinco paradigmas básicos: 1) economía de frontera, centrada en la defensa del progreso como crecimiento económico; 2) protección ambiental, fundamentalmente preocupada por despertar la conciencia frente a las situaciones ambientales y la búsqueda de conciliación entre la conservación y el crecimiento 
económico; 3) economía de los recursos, cuyo interés está en determinar la incidencia del daño ambiental en los procesos económicos y en analizar el establecimiento de sanciones de mitigación centradas bajo la premisa "el que contamina paga"; 4) ecodesarrollo, que busca armonizar la relación hombre-naturaleza desde la perspectiva de la prevención del daño ambiental como un proceso rentable, y 5) ecología profunda, que reconoce la igualdad de las especies, la promoción de la biodiversidad y la pluriculturalidad, y la autonomía biorregional.

En el marco de estas discusiones se clasificaron las formas de concebir la relación comunicación-desarrollo-medioambiente en dos vías: desde las visiones históricas y desde los agentes que promueven estos procesos.

En cuanto a las visiones históricas se establecieron tres: 1) una visión originaria de los tiempos de la guerra fría, cuando lo medioambiental se remite directamente al desarrollo rural, y la comunicación se equipara con una información al servicio de la productividad económica; 2) una visión originada en los ańos ochenta, que plantea miradas alternativas frente a los procesos de comunicación y el medioambiente: la sostenibilidad y el papel de los países del "tercer mundo" en la definición del desarrollo; 3) una visión relacionada con el desarrollo, originada en la última década del siglo XX, en la que prima el respeto por las identidades y particularidades de los pueblos a través de modernidades alternativas y el respeto por la autodeterminación, lo que se extiende a la autodefinición de lo que conciben o no como desarrollo y su relación con el medioambiente. ${ }^{1}$

Desde los agentes que generan dichos procesos de comunicación-desarrollomedioambiente, también se pensó en tres grandes tendencias que apuntan hacia la comprensión y el vínculo con perspectivas e intereses distintos: 1) una tendencia que establece la relación comunicación-desarrollo-medioambiente desde la perspectiva de la comunicación y la participación social y comunitaria; 2) una línea en la que se encuentran las propuestas desde la comunicación de organizaciones estatales,

1 Una explicación más detallada de toda la propuesta conceptual puede consultarse en el siguiente vínculo: http:// experienciascomunicacionyambiente.blogspot.com/ 
empresariales e industriales; 3) una tendencia focalizada en las agendas mediáticas y el posicionamiento del tema en la esfera pública, a través de los medios ${ }^{2}$.

\section{Diseño METODOLÓGICO}

Desde la formulación del proyecto, la investigación buscó identificar y caracterizar experiencias de comunicación y desarrollo que aportaran a la gestión y transformación social de problemáticas medioambientales en la región andina de Colombia, así como la comprensión, desde los contextos históricos y culturales, de la relación comunicación-desarrollo-medioambiente en una muestra de dichas experiencias. Por esta razón, se estructuró un diseño metodológico que incluye el cruce de estrategias cuantitativas y cualitativas, así como la reflexión complementaria entre un proceso empírico-analítico y otro histórico-hermenéutico, desde un análisis macrosocial y un análisis microsocial, respectivamente.

Por la dificultad que representaba ubicar una base de datos única que identificara las experiencias de comunicación y desarrollo sobre medioambiente en la región, el equipo vio pertinente proponer un cruce metodológico macro- y microsocial, consecuente también con los hallazgos del estado del arte, que señalaban:

Muy pocos trabajos se inscriben dentro del enfoque empírico analítico positivista. Esto sugiere la necesidad de tener en cuenta este tipo de estudios, pues en gran parte de las investigaciones se hace evidente la ausencia de un mapeo general que permita hacer inferencias y que ofrezca pistas precisas y cuantificables que sirvan de base para los análisis de tipo cualitativo (Herrera y Uruburu, 2009: 129).

El fin específico del análisis macrosocial fue mapear experiencias de comunicación y desarrollo en la región andina, a través de un instrumento de observación estructurado con base en variables de nivel ordinal y nominal (la mayoría de ellas) y cualificado con preguntas abiertas. La reflexión conceptual contribuyó a la definición de los aspectos necesarios para que el mapeo brindara una identificación y descripción

2 Para ampliar las características de cada una de estas tendencias, véase: Herrera (2009: 29 y ss.). 
general, con la mayor cantidad de pistas posibles sobre las características de las experiencias, en tres sentidos: ubicación espacio-temporal, comunicación (visión, procesos, estrategias) e implicaciones sociales (problemas ambientales, actores vinculados, procesos de participación, relación con redes, dificultades, legalidad). Dicha descripción permitió plantear criterios de selección de experiencias con miras a la realización de la etapa microsocial.

El análisis microsocial indagó por los actores, las creencias, las tradiciones, los conocimientos e imaginarios de unas comunidades frente al medioambiente, al igual que su concepción de este, sus hábitos y sus costumbres, las estrategias como técnicas, planificación y articulación de los recursos que implementaban; las concepciones que se tienen del desarrollo y la comunicación, sus lenguajes, sus procesos, los escenarios, las formas de participación, las redes y las relaciones, entre otros tantos aspectos que surgieron en el trabajo de campo. Aquí los instrumentos de observación para el trabajo de campo y los estudios de caso fueron los mismos para las tres universidades, y se definieron a partir de jornadas en las cuales se operacionalizaron los objetivos, como se explicará más adelante.

De esta forma, el desarrollo del proyecto contempló cuatro etapas o momentos: 1) etapa macrosocial, para hacer el mapeo de las experiencias, con el fin de identificarlas (cuántas y cuáles) y hacer una descripción general de esas experiencias; 2) análisis microsocial, mediante el desarrollo de diez estudios de caso, con el ánimo de comprender y profundizar las experiencias; 3) sistematización de la información y triangulación de los resultados cualitativos y cuantitativos; 4) diálogo de saberes, para socializar los resultados y hallazgos con la comunidad académica y con las comunidades de las subregiones, lo que incluyó estrategias de visibilización, construcción de redes y trabajo interactivo.

\section{Mapeo de experiencias}

La finalidad del análisis macrosocial es lograr una aproximación a la realidad sociocultural, a través de la identificación de actores, eventos y situaciones para obtener un cuadro completo del fenómeno analizado (Sandoval, 1996). Con esta 
lógica, el proceso de mapeo se desarrolló a través de la convocatoria, búsqueda e identificación de experiencias de comunicación, desarrollo y medioambiente en la región andina, para lo cual se establecieron contactos virtuales y telefónicos desde bases de datos de diferentes orígenes ${ }^{3}$, recolectadas por el equipo de investigación.

Después de un intenso trabajo de llamadas telefónicas y envío permanente de correos electrónicos, entre febrero y julio de 2009, el equipo logró identificar cerca de 250 experiencias, a las cuales se les solicitó diligenciar una encuesta. Así, se logró una muestra autogenerada de 112 casos, los cuales constituyen el cuerpo de este análisis macrosocial.

El instrumento de observación se elaboró pensando en aspectos que fueran claves para la ubicación, identificación y tipificación de las experiencias en términos generales o macrosociales. La encuesta constaba de 21 preguntas y contempló: datos específicos de la experiencia (8); preguntas sobre sus procesos y actores con opciones cerradas dicotómicas (2) y cerradas de opción múltiple (2); y preguntas abiertas sobre su origen, visión sobre el medioambiente, visión sobre comunicación, actividades, estrategias, dificultades y procesos de participación (9).

Las dificultades en la recolección de la información obligaron a la modificación del cronograma inicial, lo que significó que en lugar de dos meses se requirió hacer un trabajo de cinco. Estas dificultades se derivaron del diseño mismo del proyecto, de las condiciones administrativas institucionales y de las circunstancias externas que se fueron detectando en el camino, producto, en algunos casos, de experiencias de investigación que las comunidades han enfrentado en el pasado. Dichas dificultades constituyen un primer aprendizaje de orden metodológico muy útil para experiencias posteriores.

Las bases de datos de diferentes instituciones que sirvieron de apoyo para la recolección de la información, que requerían el uso de tecnologías de la comunicación como telefonía e internet, presentaron inconsistencias que demandaron un largo proceso

3 Se utilizaron bases de datos de radios y televisiones comunitarias, grupos juveniles, grupos sociales, comunidades de base, grupos ambientales, así como informaciones de organizaciones gubernamentales y no gubernamentales relacionadas con el tema medioambiental. La convocatoria de búsqueda de experiencias se publicó en diferentes boletines virtuales, como el de la organización Medios para la Paz. 
de depuración de los datos. El aprendizaje de este proceso demuestra que la vía de comunicación a través de la red, en términos de su diseńo, es de limitada utilidad, por lo que se estableció contacto telefónico; sin embargo, aun así no se obtuvo respuesta en, por lo menos, 140 casos. Una dificultad institucional se asocia con la disponibilidad de líneas abiertas para hacer llamadas de larga distancia o a celular, situación que demandó una gestión administrativa que prolongó más de lo previsto este proceso.

Dentro de las circunstancias externas, se cuenta con la identificación de experiencias coyunturales conformadas para proyectos puntuales, pero que en el momento de contactarlas ya no eran vigentes, por lo cual sus líderes no vieron pertinente compartir la información. En otros casos, la dificultad estuvo en los “intermediarios" entre las comunidades y los investigadores, pues aquellos obstruyeron el acceso a la información argumentando desinterés, cuando en algunas ocasiones lo que existía era una desconfianza o temor de compartir sus experiencias con las universidades, porque estas no retribuirían, de alguna manera, la cooperación y dedicación de la comunidad.

Para el proceso de sistematización, una vez concluida la recolección de las encuestas, se realizó una lectura general con el ánimo de proponer categorías inductivas. En varias jornadas de trabajo, el equipo fue puntualizando y definiendo las categorías a partir de las mismas respuestas, para orientar la posterior sistematización de la información. Desde este proceso inductivo, se establecieron las categorías de la tabla 1, donde los aspectos corresponden a las variables planteadas desde las preguntas de la encuesta, y las categorías, a posibles agrupaciones de las respuestas según sus definiciones.

Esta categorización inductiva se codificó y se tabuló para tipificar las experiencias de comunicación y desarrollo sobre medioambiente en la región andina de Colombia, a través del análisis de los datos y lecturas estadísticas de proporciones (porcentaje), de jerarquía (percentílicas), descriptivas (promedio) e inferenciales (desviación estándar, coeficiente de asimetría). Esta caracterización de las experiencias no fue producto de relaciones espurias entre los datos, sino de procedimientos que permitieron establecer percentiles de la cobertura de una variable con respecto a las otras, facilitando así su jerarquización y permitiendo establecer referentes para comparaciones ulteriores con experiencias de otras regiones del país. 
Tabla 1. Categorías inductivas

\begin{tabular}{|c|c|c|}
\hline Aspecto & Categoría & Definición \\
\hline \multirow{3}{*}{$\begin{array}{l}\text { Origen de la } \\
\text { experiencia } \\
\text { (agente gestor } \\
\text { inicial) }\end{array}$} & Organizaciones sociales & $\begin{array}{l}\text { Experiencias propuestas por habitantes y } \\
\text { líderes de la comunidad para abordar alguna } \\
\text { problemática ambiental particular, o varias de una } \\
\text { localidad o región, cuya motivación posibilita la } \\
\text { contextualización natural, social y cultural, a la vez } \\
\text { que genera procesos de solución. }\end{array}$ \\
\hline & Institucional & $\begin{array}{l}\text { Experiencias cuyo proceso se gesta en una o varias } \\
\text { instituciones públicas o privadas en el contexto } \\
\text { de sus intereses de responsabilidad social o de } \\
\text { comunicación externa (convocatorias, convenios, } \\
\text { campańas y capacitaciones). }\end{array}$ \\
\hline & Mediático & $\begin{array}{l}\text { Experiencias originadas en los medios locales y } \\
\text { regionales, producto del deseo de poner en la agenda } \\
\text { mediática el tema ambiental. }\end{array}$ \\
\hline \multirow{3}{*}{$\begin{array}{l}\text { Problemáticas } \\
\text { ambientales }\end{array}$} & $\begin{array}{l}\text { Protección y manejo de } \\
\text { recursos naturales }\end{array}$ & $\begin{array}{l}\text { Actividades relacionadas con la manera en que } \\
\text { se trabaja sobre el cuidado y manejo de recursos } \\
\text { específicos, como el agua, el suelo y el aire. }\end{array}$ \\
\hline & $\begin{array}{l}\text { Concientización, } \\
\text { sensibilización y } \\
\text { empoderamiento }\end{array}$ & $\begin{array}{l}\text { Acciones en pro de visibilizar o generar cambios de } \\
\text { actitudes y valores solidarios significativos sobre las } \\
\text { situaciones ambientales vividas, para favorecer la } \\
\text { búsqueda de soluciones. }\end{array}$ \\
\hline & Territorio & $\begin{array}{l}\text { Dinámicas de recuperación y fortalecimiento del } \\
\text { entorno local y regional, a partir de las diferentes } \\
\text { problemáticas ambientales en un espacio y tiempo } \\
\text { concretos, donde se reconoce de manera específica } \\
\text { las condiciones sociales, económicas, políticas o } \\
\text { culturales propias del contexto. }\end{array}$ \\
\hline \multirow{3}{*}{ Tipo de redes } & Ambientales & $\begin{array}{l}\text { Experiencias en las que participan actores (sujetos } \\
\text { y entidades locales, regionales y nacionales) } \\
\text { interesados en la resolución de problemáticas } \\
\text { ambientales y agrícolas. }\end{array}$ \\
\hline & Sociales y comunitarias & $\begin{array}{l}\text { Experiencias desarrolladas en el marco de un } \\
\text { trabajo conjunto, vinculadas por su carácter social y } \\
\text { comunitario. }\end{array}$ \\
\hline & Comunicativas & $\begin{array}{l}\text { Actividades de interacción con otros grupos } \\
\text { u organizaciones que desarrollan procesos de } \\
\text { comunicación, medios informativos comunitarios, } \\
\text { mesas de trabajo, etc. }\end{array}$ \\
\hline
\end{tabular}




\begin{tabular}{|c|c|c|}
\hline Aspecto & Categoría & Definición \\
\hline \multirow{4}{*}{$\begin{array}{l}\text { Estrategias } \\
\text { comunicativas }\end{array}$} & Mediáticas & $\begin{array}{l}\text { Implementación de medios en el desarrollo } \\
\text { de la experiencia; uso de la radio comunitaria, } \\
\text { audiovisuales, prensa, internet, carteles, periódicos } \\
\text { murales, etc. }\end{array}$ \\
\hline & Educación ambiental & $\begin{array}{l}\text { Acciones educativas y de capacitación que } \\
\text { contribuyan a comprender las relaciones de } \\
\text { interdependencia con el entorno, a partir de un } \\
\text { conocimiento reflexivo, crítico y aplicado desde } \\
\text { su realidad biofísica, social, política, económica } \\
\text { y cultural, a fin de generar un cambio de actitud, } \\
\text { valoración y respeto por su ambiente. }\end{array}$ \\
\hline & Investigativas & $\begin{array}{l}\text { Estrategias de observación y recolección de } \\
\text { información pertinente para la experiencia; registro } \\
\text { de la memoria audiovisual; búsqueda de testimonios } \\
\text { y personajes que puedan aportar a la experiencia; } \\
\text { reinterpretación de experiencias metodológicas; } \\
\text { reconocimiento de saberes provenientes de otras } \\
\text { lógicas (indígenas), y recuperación de la historia } \\
\text { ambiental de los espacios. }\end{array}$ \\
\hline & Lúdico-artísticas & $\begin{array}{l}\text { Actividades de socialización de las temáticas } \\
\text { ambientales, a través de acciones culturales y } \\
\text { artísticas (canto, danza, teatro, etc.). }\end{array}$ \\
\hline \multirow{4}{*}{$\begin{array}{l}\text { Actividades } \\
\text { participativas }\end{array}$} & Visibilización externa & $\begin{array}{l}\text { Participación en concursos, encuentros, ferias, foros, } \\
\text { seminarios y festivales, en los cuales se comparte la } \\
\text { experiencia con otros y esta se hace visible ante la } \\
\text { sociedad. }\end{array}$ \\
\hline & Sensibilización & $\begin{array}{l}\text { Acciones tendientes a concienciar e influenciar a las } \\
\text { comunidades, para invitarlas a recapacitar frente a } \\
\text { la situación ambiental; desarrollo de campañas para } \\
\text { generar conciencia sobre el problema. }\end{array}$ \\
\hline & Capacitación & $\begin{array}{l}\text { Actividades de formación y capacitación formal } \\
\text { e informal en las comunidades; realización de } \\
\text { talleres, caminatas y actividades lúdico-pedagógicas; } \\
\text { vinculación de instituciones educativas a la } \\
\text { experiencia. }\end{array}$ \\
\hline & Diálogos & $\begin{array}{l}\text { Estrategias de integración y realización de tertulias, } \\
\text { asambleas, reuniones informativas y encuentros, } \\
\text { para tratar las temáticas ambientales de la localidad } \\
\text { o la región. }\end{array}$ \\
\hline
\end{tabular}




\begin{tabular}{|c|l|l|}
\hline \multicolumn{1}{|c|}{ Aspecto } & \multicolumn{1}{|c|}{ Categoría } & \multicolumn{1}{c|}{ Definición } \\
\hline \multirow{5}{*}{$\begin{array}{l}\text { Dificultades u } \\
\text { obstáculos }\end{array}$} & Institucionales & $\begin{array}{l}\text { Falta de recursos y apoyo económico; las } \\
\text { experiencias no son autosostenibles. }\end{array}$ \\
\cline { 2 - 3 } & Responsabilidad ambiental & $\begin{array}{l}\text { No hay credibilidad en las instituciones (no } \\
\text { estatales); hace falta apoyo por parte de estas } \\
\text { organizaciones en las experiencias; ciertos } \\
\text { compromisos se establecen y luego se incumplen. }\end{array}$ \\
\cline { 2 - 4 } & $\begin{array}{l}\text { Desinterés por parte de actores de las comunidades } \\
\text { e instituciones para trabajar el tema; falta de } \\
\text { conciencia por asumir la responsabilidad social } \\
\text { de cuidar el entorno; carencia de agendas de } \\
\text { información educativa, y acceso reducido a la } \\
\text { información. }\end{array}$ \\
\cline { 2 - 4 } & Apoyo estatal & $\begin{array}{l}\text { Indiferencia por parte de autoridades o instituciones } \\
\text { estatales frente a problemáticas ambientales locales y } \\
\text { regionales. }\end{array}$ \\
\cline { 2 - 4 } & Respaldo técnico & $\begin{array}{l}\text { Desconocimiento de manejo adecuado de } \\
\text { recursos; falta de acompañamiento profesional } \\
\text { (comunicativo o medioambiental) adecuado a las } \\
\text { iniciativas. }\end{array}$ \\
\hline
\end{tabular}

Fuente: eleaboración de los autores.

Por otro lado, la pregunta por la visión de la comunicación presentó, para el equipo de trabajo, una dificultad y un reto en el proceso de categorización, a causa de la tendencia de los investigadores a proponer categorías provenientes de las teorías de la comunicación sin tener en cuenta la construcción de las comunidades. Este proceso se resolvió inductivamente a través del software Atlas.ti, para conocer el sentido que las experiencias atribuyen a la comunicación, identificando seis categorías emergentes (Coulon, 1988) producto de las interpretaciones a la pregunta abierta "Para ustedes, ¿qué es la comunicación?”.

Los hallazgos en esta etapa de la investigación fueron fundamentales para diseñar el análisis microsocial. Inicialmente se pensó en tomar solo experiencias con origen en organizaciones comunitarias, pero a partir de los resultados del mapeo se escogieron experiencias (tabla 2) con más de cuatro años de labor, ubicadas en diferentes subregiones de la región andina, con un aspecto relevante respecto a la vinculación y participación de diversos actores sociales, la relación con redes y la incidencia social de su trabajo mediante la movilización de la comunidad. 
Tabla 2. Experiencias seleccionadas

\begin{tabular}{|c|c|c|c|c|}
\hline Experiencia & Tiempo & $\begin{array}{c}\text { Departamento } \\
\text { y municipio }\end{array}$ & Subregión & Aspecto relevante \\
\hline $\begin{array}{l}\text { Sibaté, } 12.000 \\
\text { años de historia }\end{array}$ & 20 años & $\begin{array}{l}\text { Cundinamarca, } \\
\text { Sibaté }\end{array}$ & $\begin{array}{l}\text { Altiplano } \\
\text { cundiboyacense }\end{array}$ & $\begin{array}{l}\text { Procesos de reconstrucción y } \\
\text { recuperación de memoria histórica } \\
\text { de largo aliento }\end{array}$ \\
\hline $\begin{array}{l}\text { Programa de } \\
\text { radio Magazin } \\
\text { Agenda } \\
\text { Ambiental }\end{array}$ & 7 ańos & Huila, Pitalito & $\begin{array}{l}\text { Macizo } \\
\text { colombiano }\end{array}$ & $\begin{array}{l}\text { Desarrollo de acciones educativas } \\
\text { y participativas que evidencian la } \\
\text { movilización de la comunidad }\end{array}$ \\
\hline $\begin{array}{l}\text { Escuela de } \\
\text { Comunicación } \\
\text { Minga del Sol }\end{array}$ & 4 años & Huila, Neiva & Alto Magdalena & $\begin{array}{l}\text { Trabajo con grupos juveniles de } \\
\text { comunidades vulnerables }\end{array}$ \\
\hline $\begin{array}{l}\text { Federación de } \\
\text { Prosumidores } \\
\text { Agroecológicos }\end{array}$ & 6 años & $\begin{array}{l}\text { Boyacá, } \\
\text { Tibasosa }\end{array}$ & $\begin{array}{l}\text { Altiplano } \\
\text { cundiboyacense }\end{array}$ & $\begin{array}{l}\text { Trabajo comunitario en red con } \\
\text { comunidades campesinas }\end{array}$ \\
\hline $\begin{array}{l}\text { Corporación } \\
\text { Ciudad Rural }\end{array}$ & 10 años & $\begin{array}{l}\text { Antioquia, } \\
\text { San Antonio } \\
\text { del Prado } \\
\text { (corregimiento } \\
\text { de Medellín) } \\
\end{array}$ & $\begin{array}{l}\text { Montañas } \\
\text { antioqueñas }\end{array}$ & $\begin{array}{l}\text { Rescate de la ruralidad con } \\
\text { integración de diferentes medios }\end{array}$ \\
\hline $\begin{array}{l}\text { Fundación } \\
\text { Planeta Azul y } \\
\text { Vida }\end{array}$ & 5 años & $\begin{array}{l}\text { Cauca, } \\
\text { Popayán }\end{array}$ & $\begin{array}{l}\text { Altiplano de } \\
\text { Popayán y } \\
\text { la cordillera } \\
\text { central } \\
\text { meridional }\end{array}$ & $\begin{array}{l}\text { Trabajo en red con radios y } \\
\text { canales de televisión comunitaria, } \\
\text { como Balboa Estéreo, Piendamó } \\
\text { Estéreo, Voz de Belalcázar y } \\
\text { Ondas de Ibagué; canales locales } \\
\text { en Popayán, Piendamó, Santander } \\
\text { de Quilichao y El Bordo }\end{array}$ \\
\hline $\begin{array}{l}\text { Cátedra de } \\
\text { Educación } \\
\text { Ambiental } \\
\text { y Centro de } \\
\text { Investigaciones } \\
\text { Bioecológicas }\end{array}$ & 5 años & $\begin{array}{l}\text { Valle del } \\
\text { Cauca, } \\
\text { Guadalajara de } \\
\text { Buga }\end{array}$ & Valle del Cauca & $\begin{array}{l}\text { Educación ambiental con } \\
\text { procesos de investigación. } \\
\text { Recibió el Premio Nacional } \\
\text { al mérito científico en el área } \\
\text { Divulgación de la Ciencia, } \\
\text { otorgado por la Asociación } \\
\text { Colombiana para el Avance de la } \\
\text { Ciencia }\end{array}$ \\
\hline $\begin{array}{l}\text { Emisora San } \\
\text { Vicente Estéreo }\end{array}$ & 10 años & \begin{tabular}{|l|} 
Santander, \\
San Vicente de \\
Chucurí
\end{tabular} & $\begin{array}{l}\text { Magdalena } \\
\text { Medio }\end{array}$ & $\begin{array}{l}\text { Miembro de la Asociación Red de } \\
\text { Emisoras del Magdalena Medio } \\
\text { (Aredmag) }\end{array}$ \\
\hline
\end{tabular}




\begin{tabular}{|l|l|l|l|l|}
\hline \multicolumn{1}{|c|}{ Experiencia } & Tiempo & $\begin{array}{l}\text { Departamento } \\
\text { y municipio }\end{array}$ & Subregión & \multicolumn{1}{c|}{ Aspecto relevante } \\
\hline $\begin{array}{l}\text { Programa de } \\
\text { radio Radioam- } \\
\text { bientalitos }\end{array}$ & 10 años & $\begin{array}{l}\text { Norte de } \\
\text { Santander }\end{array}$ & $\begin{array}{l}\text { Macizo de San } \\
\text { Turbán }\end{array}$ & $\begin{array}{l}\text { Trabajo con nińos de colegios en } \\
\text { la emisora comunitaria Nuestra } \\
\text { Señora de las Mercedes de } \\
\text { Mutiscua }\end{array}$ \\
\hline $\begin{array}{l}\text { Asociación } \\
\text { Amigos de la } \\
\text { Cultura y la } \\
\text { Comunicación } \\
\text { de Montenegro }\end{array}$ & 10 años & $\begin{array}{l}\text { Quindío, } \\
\text { Montenegro }\end{array}$ & $\begin{array}{l}\text { Cordillera } \\
\text { central } \\
\text { meridional, } \\
\text { zona del eje } \\
\text { cafetero }\end{array}$ & $\begin{array}{l}\text { Emisora comunitaria Montenegro } \\
\text { Estéreo, con acciones } \\
\text { participativas de reforestación y } \\
\text { preservación de especies }\end{array}$ \\
\hline
\end{tabular}

Fuente: eleaboración de los autores.

\section{Estudios de caso}

Una vez culminada la fase de mapeo, se continuó con el análisis microsocial, que pretendió comprender las visiones sobre comunicación y desarrollo que inspiran estas experiencias de gestión medio ambiental. Esto se realizó de una manera más detallada y estableció lecturas intermedias y profundas sobre las prácticas y redes sociales construidas en torno a estos procesos de comunicación y desarrollo sobre medio ambiente en la región andina de Colombia.

El método central fue el trabajo etnográfico, apoyado fundamentalmente en las técnicas cualitativas de investigación, donde se presenta un contacto directo con las comunidades. Así, se trasciende al trabajo de campo, "que tiene como eje fundamental la observación, pues este es el instrumento por excelencia para aprehender la totalidad de lo social que se manifiesta en la experiencia" (Tezanos, 1998: 22).

En primera instancia, el equipo de investigadores trabajó en la operacionalización de los objetivos, es decir, a partir de los objetivos específicos identificar conceptos del campo temático, las unidades de análisis y, luego, las categorías observacionales, para lo cual se tomó como punto de partida los hallazgos del mapeo que explicitaran los aspectos sobre los cuales se debía orientar la experiencia etnográfica (tabla 3). 
Tabla 3. Ejemplo de operacionalización de objetivos

\begin{tabular}{|c|c|c|c|}
\hline Objetivo & $\begin{array}{l}\text { Campo } \\
\text { temático }\end{array}$ & $\begin{array}{c}\text { Categorías } \\
\text { observacionales }\end{array}$ & Aspectos \\
\hline \multirow{7}{*}{$\begin{array}{l}\text { Reconocer las } \\
\text { visiones sobre } \\
\text { comunicación } \\
\text { que subyacen } \\
\text { a los procesos } \\
\text { y estrategias } \\
\text { comunicativas que } \\
\text { adelantan estas } \\
\text { comunidades }\end{array}$} & \multirow{7}{*}{ Comunicación } & $\begin{array}{l}\text { Concepción de } \\
\text { comunicación }\end{array}$ & $\begin{array}{l}\text { Visiones, significaciones, sentidos sobre } \\
\text { comunicación }\end{array}$ \\
\hline & & $\begin{array}{l}\text { Concepción } \\
\text { de medios de } \\
\text { comunicación }\end{array}$ & $\begin{array}{l}\text { Visiones, significaciones, sentidos sobre los } \\
\text { medios }\end{array}$ \\
\hline & & Procesos & $\begin{array}{l}\text { Descripción de rutinas y dinámicas de } \\
\text { producción comunicativa }\end{array}$ \\
\hline & & Lenguajes & $\begin{array}{l}\text { Forma de expresión verbal, no verbal, } \\
\text { mediáticas, artísticas, narrativas y } \\
\text { construcción de sentido }\end{array}$ \\
\hline & & Estrategias & $\begin{array}{l}\text { Planificación, técnicas utilizadas en } \\
\text { términos comunicativos }\end{array}$ \\
\hline & & Propósitos & $\begin{array}{l}\text { Razones, fines e intenciones } \\
\text { comunicacionales }\end{array}$ \\
\hline & & Escenarios & $\begin{array}{l}\text { Espacios de liberación mediáticos y no } \\
\text { mediáticos }\end{array}$ \\
\hline
\end{tabular}

Fuente: eleaboración de los autores.

Desde este proceso se establecieron cinco categorías axiales: comunicación, medio ambiente, desarrollo, incidencia y agentes, que se constituyeron en el primer momento para la escogencia de unidades de análisis "a través de un proceso deductivo, es decir, se selecciona qué se va a observar en función de investigaciones pasadas, hipótesis previas de investigación o unidades anteriormente observadas" (Rodríguez, Gil y García, 2002: 163).

Estas categorías orientaron la definición y aplicación de técnicas e instrumentos para el trabajo de campo: observación participante y no participante con registro en diario de campo, entrevistas en profundidad y entrevistas colectivas, relato de vida sobre el líder de la experiencia y talleres con grupos de la comunidad definidos por los mismos líderes de cada experiencia.

En lo concerniente a las directrices para la recolección de la información, se tenían lineamientos desde el trabajo analítico desarrollado con los objetivos: tanto los asistentes como investigadores deberían llevar un diario de campo detallado con las 
respectivas descripciones de sujetos, acontecimientos, tiempos y espacios en la experiencia de comunicación. En primera instancia, el diario contemplaba una narración abierta del caso mediante descripciones que pretendían registrar, de manera amplia, todo lo que podía parecer de interés para el objeto de estudio, y luego una breve interpretación desde cada una de las cinco categorías definidas. Esto permitía que la observación y su registro no quedaran cerrados a los elementos de campo temático, sino que sirvieran de guía para la observación.

La experiencia evidenció que dichos instrumentos permitieron guiar a los asistentes de investigación en el cómo registrar, mediante el ordenamiento de los procesos de generación de conocimiento de los asistentes de investigación y de los investigadores propiamente dichos. Igualmente, la experiencia hizo posible reflexionar sobre el qué registrar, lo que logró acotar las observaciones para aprovechar la profundidad y pertinencia de los registros.

Por otra parte, se programaron cuatro talleres: uno de cartografía social para diagnosticar, de manera participativa con la comunidad, las problemáticas medioambientales; otro para aproximarse a las visiones de desarrollo; y en los otros dos talleres se atendieron las sugerencias que hicieron los líderes de las experiencias, pues la idea era responder a algunas necesidades teórico-prácticas específicas. De esta manera, se buscó que en los espacios de los talleres se permitiera un contacto participativo de los agentes vinculados a la experiencia.

Además, las técnicas de investigación incluyeron entrevistas en profundidad tanto de los líderes de cada experiencia como de otros agentes involucrados en el trabajo de comunicación en medio ambiente. Esta técnica ofrece la ventaja de la interacción cara a cara "con sus componentes emocionales y empáticos, facilita la fidelidad del registro y aumenta el grado de comprensión e interpretación de los datos" (Gaitán y Piñuel, 1998: 111). Asimismo, este tipo de entrevista permite profundizar en las respuestas, pedir ampliación o explicación al entrevistado, pero también se complementa la información a través de la observación no solo del contexto, si esta tiene lugar en un espacio cotidiano del entrevistado, sino de la combinación del lenguaje no verbal, que se contrasta con la narración verbal para mirar si el uno contradice a la otra o la reitera (Gaitán y Piñuel, 1988). 
La pauta para la selección de la muestra de sujetos para entrevistar fue su vinculación con la experiencia de comunicación. En este sentido, no había una muestra definida previamente, sino que la selección se hizo en el transcurso del trabajo de campo; por esta razón, cada caso reportó diferente número de entrevistados.

Finalmente, la apuesta metodológica concluyó con un relato de vida de los líderes principales de cada experiencia, pues desde los diferentes momentos de acercamiento se apreció la dedicación, constancia e incidencia del trabajo de estas personas en cada una de sus comunidades.

De acuerdo con las exigencias de la investigación, se buscó que el contacto con la experiencia fuera el más prolongado posible, de ahí que inicialmente se pensó en un trabajo de campo de sesenta días que se haría en dos momentos diferentes, cada uno de treinta días, con un intervalo de un mes, para, en el primer momento, tener una visión general de la experiencia que se profundizaría en el segundo viaje al lugar de la experiencia; sin embargo, la revisión de los costos de dos viajes en estas condiciones mostró un incremento que superó el presupuesto en lo referente a trabajo de campo, razón por la cual se estableció que este se haría en la mitad del tiempo, es decir, treinta días de permanencia en cada uno de los diez sitios ya referidos.

Así lo hizo el equipo de investigación de la Universidad Santo Tomás, que pudo enviar, durante treinta días, a los asistentes de investigación al lugar de la experiencia, y los investigadores permanecieron allí por una semana. La Universidad Nacional Abierta y a Distancia, por condicionamientos académicoadministrativos, realizó su trabajo etnográfico el mismo tiempo, pero en dos momentos diferentes, y no contaron con asistentes de investigación. Por su parte, el equipo de investigación de la Corporación Universitaria Minuto de Dios, que sí contó con asistentes, ejecutó esta etapa de trabajo de campo en quince días, compensados con el hecho de tener, durante todo el tiempo, dos personas recolectando la información en cada lugar. Durante este tiempo, la comunicación entre investigadores fue constante para enriquecer las experiencias de un equipo de investigación con las de los otros, al mismo tiempo que se compartieron angustias pero también importantes hallazgos. 
Al concluir el trabajo etnográfico, el equipo de investigación logró reunir una buena cantidad y calidad de registros fotográficos, sonoros e impresos pertinentes para el análisis hermenéutico que se hizo con apoyo del software informático Atlas.ti, desde las cinco categorías axiales (agentes, comunicación, medio ambiente, incidencia y desarrollo); no obstante, el hecho de haber recolectado tal volumen de información representó una riqueza para la investigación, pero al mismo tiempo un reto para su sistematización, teniendo en cuenta, precisamente, el tiempo de permanencia en cada lugar y las técnicas de investigación de la estrategia metodológica.

Este análisis partió, para cada uno de los casos, de la interpretación individual desde los campos temáticos; análisis compuesto por las categorías observacionales y la emergencia de otras nuevas, con lo que se desarrolló el segundo momento de selección de unidades de análisis, a saber: "La selección mediante un proceso inductivo, a través del cual extraemos unidades de análisis más definidas y ajustadas al propio acontecimiento observado, precisamente a partir de los propios registros” (Rodríguez, Gil y García, 2002: 163).

Una vez realizado este proceso de análisis e interpretación de los estudios de caso, del cual se derivan informes de casos individuales, se dio paso a un análisis transversal por campo temático, a través de lo cual se mostraron los resultados en conjunto de las concepciones que dichas comunidades tienen sobre el desarrollo; las visiones sobre comunicación que subyacen a los procesos y estrategias comunicativas; la relación de las comunidades con el medio ambiente; las preocupaciones medioambientales que inspiran estos proyectos, y las implicaciones e incidencia que tienen estos procesos de comunicación y desarrollo.

\section{REFLEXIONES PARA EL APRENDIZAJE}

\section{Cruce de metodologías}

La apuesta por una doble metodología amplía los tiempos básicos para el desarrollo de los proyectos previstos a un año; sin embargo, este ejercicio ratifica la pertinencia del cruce y el vínculo de enfoques investigativos, porque la combinación de análisis 
macro y micro proporciona una visión más completa de la complejidad social, con datos nomotéticos sobre tendencias y rasgos generales y datos ideográficos que acercan a características específicas, particulares, que permiten entender matices a partir de aspectos históricos y culturales (Del Río, 1996).

Esta combinación o triangulación posibilita el reconocimiento de elementos objetivos y subjetivos de la realidad, asumiendo la propuesta de Bonilla y Rodríguez (1997):

El reto del investigador social debería ser el desarrollo de su capacidad analítica y de sus conocimientos para emplear los métodos de manera integrada que le posibiliten comprender la realidad social en sus dos dimensiones esenciales, a saber: la cuantitativa y la cualitativa.

En este sentido, el mapeo proporcionó una contextualización para ubicar los casos dentro de la aproximación a una posible tipificación de las experiencias; a su vez, los estudios de caso permiten comprender, más allá de la tipificación cuantitativa, las vivencias cotidianas de la relación comunicación y desarrollo sobre medioambiente desde la perspectiva de los sujetos.

\section{Manejo y tratamiento de la información}

La primera reflexión para compartir con los lectores en torno al tratamiento de los datos se relaciona con el diseńo de los instrumentos de observación de la investigación, pues en su estructura se buscaron subsanar los constantes vacíos que se presentan entre la recolección de los datos y su sistematización. Este momento de diseńo del instrumento es concebido como una subfase del proceso de investigación que suele menospreciarse en los proyectos.

Por lo anterior, lo que deja esta experiencia es que al ser prevista esta subfase, es posible potenciar significativamente los análisis e interpretaciones ulteriores, puesto que las estrategias implementadas guiaron con éxito la fase de recolección de datos durante el trabajo de campo; posteriormente, también coadyuvaron con la optimización de la sistematización o segmentación inicial de los registros de campo. 
Pero esta subfase no terminó en estas posibilidades procedimentales, sino que también disminuyó la distancia que caracteriza a los datos recolectados de fuentes secundarias entre el analista de la información y el etnógrafo, quienes no suelen ser los mismos por razones generalmente exógenas a los propios investigadores. Esta distancia se devela en el inevitable filtro que dejan los otros participantes del proceso investigativo, como los auxiliares de investigación, los representantes de las experiencias y, por supuesto, los participantes de las comunidades.

No obstante, desde la formulación de los objetivos, las posibles consecuencias de esta distancia fueron contempladas durante las etapas de definición operativa de las categorías, pero sobre todo fueron pensadas con base en la concepción del análisis de los registros de trabajo de campo como una posibilidad para reelaborar lecturas y reconstruir los datos cualitativos (Hammersley y Atkinson, 2006), puesto que la mayor bondad de los datos cualitativos es su constante construcción.

Empero, para hacer justicia a la realidad de los procesos investigativos, no todo fue tan diáfano como se describe; a algunos de los asistentes, pese a su compenetración con el proyecto de investigación, se les presentaron dificultades (menores a las que emergen en investigaciones en las que no se atiende la subfase mencionada) como el ingente cúmulo de información, sin orden aparente, sobre todo para su análisis, porque su procesamiento sobrepasa aun las posibilidades organizacionales del software de apoyo y las condiciones materiales de los investigadores.

\section{Experimentación y descubrimiento}

Desde la perspectiva del instrumento implementado en el mapeo social, debe señalarse que la inscripción de las encuestas en el paradigma empírico-analítico no demanda necesariamente de un análisis y de unas lecturas exclusivamente estadístico-matemáticas, si se observa desde el punto de vista de los actuales discursos sobre la investigación. Por el contrario, lo que se evidenció en el instrumento diseñado es que al llegar a preguntas abiertas imprescindibles en el sustento epistemológico del proyecto, fue necesario recurrir a varios procesos para analizar partes del mismo instrumento, en razón de la diversidad de tipos de variables mencionadas y del 
sustancial número de preguntas abiertas que lo constituyeron; preguntas sobre las cuales difícilmente puede saberse con anterioridad un camino para su sistematización. El experimento sirvió para descubrir las bondades transversales de los métodos de análisis, al franquear los límites que imponen las técnicas y los instrumentos de investigación.

Una de las preguntas importantes fue la que indagó por el sentido de la comunicación para las 112 experiencias de la región andina. Para abordar su interpretación, primero el equipo debió deshacerse de los paradigmas de la comunicación, parte constitutiva del habitus de los investigadores, para permitir que de la autoorganización de la información se pudiera develar la acepción de comunicación que han elaborado las experiencias encuestadas. Posteriormente, se implementó un análisis booleano conjuntivo, con base en categorías emergentes, producto del método inductivo, que se asociaron con categorías a priori como las que definen el origen de las experiencias (organización social, institucional o mediática).

Este aprendizaje culminó con el descubrimiento de las bondades y las posibles interacciones entre diversos métodos analíticos y adaptaciones en los instrumentos de registro, con lo cual las concepciones del grupo de investigación se alejaron de las fronteras que establecen las definiciones sobre diseños metodológicos o, incluso, más importante, sobre la concepción de comunicación.

\section{Usuarios informáticos: apropiación del Atlas.ti}

La apropiación tecnológica previa a todo el trabajo de sistematización e interpretación surgió por el requerimiento técnico de profundizar en el manejo del programa informático para análisis de datos cualitativos Atlas.ti. Dicha apropiación comenzó con la más típica de las inquietudes de los advenedizos al campo de la investigación, con apoyo de programas informáticos (Carvajal, 2002), que puede resumirse en el reconocimiento de los alcances del programa. Lo primero que debió establecerse enfáticamente fue que el software es tan solo una herramienta como lo fueron - y aún lo son- el lápiz, el papel, los colores y las fichas clasificatorias de antaño. 
La práctica del conocimiento y reconocimiento del programa Atlas.ti durante la experiencia de capacitación permite identificar las disposiciones de los investigadores, desde diferentes posturas. Siguiendo la perspectiva de Valles (2002), podría señalarse que durante las primeras sesiones, la mayoría del grupo correspondía a tecnoentusiastas, es decir, investigadores que venían de prácticas manuales de análisis de datos cualitativos, pero que pasaron a las prácticas asistidas por computador de una manera profundamente emotiva, sufriendo lo que se denomina una tecnotransición. Sin embargo, en algunos momentos se escuchaban las exclamaciones de otro subgrupo de asistentes, los tecnoarrepentidos, aquellos investigadores que fueron usuarios, en el pasado, de programas informáticos para analizar sus datos cualitativos, pero que ahora retomaron las prácticas manuales porque no tuvieron éxito con las utilidades computacionales y, de hecho, se lamentan por haberlo intentado.

Otro grupo identificable de investigadores fue el de los tecnovanguardistas, que han sufrido la postransición; es decir, son investigadores que comenzaron a trabajar y seguirán trabajando con programas informáticos en sus procesos investigativos. Evidentemente, al tratarse el tema de la aproximación de un usuario a la informática, deben contemplarse sus características etarias y sus trayectorias profesionales, pues los programas informáticos para análisis cualitativo, o Computing Assisted Qualitative Data Analysis Software (CAQDAS), como se conocen por sus siglas en inglés, solo llevan treinta años en el campo científico, y las olas de la "revolución metodológica" continúan arremetiendo en la actualidad, cada vez con más fuerza, dado el vertiginoso crecimiento de la tecnología.

Debe resaltarse que lo más importante de esta tipologización de los asistentes, basada en la capacitación brindada por un investigador del mismo equipo interinstitucional con amplia experiencia en el programa - es decir, por un tecnovanguardista, en términos de Valles (2002)_, es que en la práctica cada investigador logró conjugar sus habilidades pasadas de análisis de datos cualitativos, basadas en estrategias eminentemente manuales, con la adopción de algunas ventajas ofrecidas por el Atlas.ti, particularmente en la sistematización.

A manera de ejemplo, algunos investigadores apoyaron manualmente sus análisis sobre los reportes del procesamiento que ofrece el programa, o generaron sus 
interpretaciones sobre la base de la representación gráfica de las categorías. En general, se articularon ambas posibilidades, manuales y tecnológicas, para dar cuenta de la interacción de todos los atributos durante los análisis categoriales. Esto, por supuesto, no dejó en riesgo la integridad del análisis e interpretaciones de los datos, porque, por fortuna, los computadores no tienen la facultad de razonar, así que los investigadores continúan siendo los analistas de la información, actividad que se despliega sobre todos los momentos de los proyectos de investigación (Valles, 2005) ${ }^{4}$ y hace parte constitutiva del capital teórico y metodológico de los investigadores.

Esto es muy importante para la integridad del proyecto y de sus supuestos epistemológicos y éticos, dado que, como puede observarse, no se ajustaron los objetivos del proyecto de investigación a la herramienta informática disponible, lo cual ha sido una práctica común en el auge de los programas informáticos para análisis de datos cualitativos en la región (Carvajal, 2002).

Contrario a la percepción generalizada, el lugar del Atlas.ti fue el de una herramienta de apoyo durante la investigación, papel que cumple cualquier otro tipo de herramienta que posibilite a los investigadores, en la práctica, dar cuenta de sus logros, descubrimientos y aportes al campo científico.

\section{Comunidad de aprendizaje}

Sobre la base de los aprendizajes del grupo de investigación, se destaca que las apuestas metodológicas se convierten en el instrumento comunicante entre el que conoce, el que es conocido y lo que es conocido, de allí que estas se muestren como el lenguaje para interrogar y conocer la realidad en un primer momento, para luego traducirla, recrearla y darla a conocer.

4 Valles (2005) considera que esta actividad, a la cual adjetiva como omnipresente, se produce desde la formulación del problema de investigación y de algunas consideraciones sobre el diseńo metodológico (análisis proyectado), pasando por la fase del trabajo de campo (análisis preliminares), hasta finalizar el proyecto, cuando se escribe y presenta el informe final de investigación (análisis intenso final). 
En este sentido, las distintas apuestas colectivas desde la primera versión de este proyecto, así como las ulteriores discusiones conceptuales en torno a la comunicación, el desarrollo y el medio ambiente, hicieron del grupo de investigación interinstitucional una comunidad de práctica, una red de práctica (Wenger, 2004) o, para señalar un concepto más adecuado, un sistema social de aprendizaje (Wenger, 2006).

Sistema social de aprendizaje es un concepto que alude a aquellos grupos de personas que comparten un interés por algo que hacen, y que aprenden cómo hacerlo mejor a través de su constante interacción (Wenger, 1998). Por ello, los individuos que conforman estas comunidades llegan a tomar la responsabilidad de administrar el conocimiento que necesitan; de hecho, se estructuran para ello, permitiendo la circulación de información, alimentación y explicitación analítica del conocimiento. Esta explicitación hace más asequible el conocimiento en determinados objetivos prácticos y, además, no tiene limitaciones estructurales ni formales para poder existir, con lo cual se beneficia a cualquier individuo y se amplían los impactos.

En este contexto, el trabajo de los integrantes del grupo de investigación interinstitucional ha generado una red de conocimiento que ha permitido el logro de los objetivos de investigación. Con ello, los investigadores se protegen de los problemas institucionales caracterizados por tramitologías y dilaciones, aúnan esfuerzos profesionales y personales y, a su vez, optimizan los recursos personales e institucionales. Una red a salvo de las falacias informacionales y de los discursos unidireccionales acerca de las redes de conocimiento.

Esto surgió desde la investigación "Estado del arte de la investigación en comunicación y desarrollo en Colombia, 2002-2006", pues se fue consolidando el grupo de estudio a partir de la apertura a los diversos intereses de los investigadores, que van desde intereses por el nivel o grado de participación de las organizaciones sociales en este tipo de experiencias comunicativas, pasando por las revisiones teóricas exhaustivas de las relaciones entre comunicación y desarrollo, hasta llegar a la reflexividad como concepto y práctica transversal al proyecto de investigación.

En síntesis, el grupo de investigación interinstitucional devino en una comunidad de aprendizaje que ha desarrollado el proyecto de investigación haciendo caso omiso a 
las restricciones formales de los convenios interinstitucionales. Así, un proyecto de investigación basado en una comunidad de aprendizaje siempre tendrá un impacto de mayor alcance que los constituidos con base en formalismos, pues estos terminan cuando los objetivos de los proyectos son cumplidos, mientras que los primeros permanecen en la medida en que sus investigadores, en un trabajo colectivo, mantengan vivo ese vínculo del placer por develar aquello que hay más allá de lo evidente.

\section{REFERENCIAS}

Bessette, G. (2004). Facilitar el diálogo, el aprendizaje y la participación para el manejo de los recursos naturales. En Comunicación y desarrollo sostenible. Selección de artículos de la 9. "Mesa Redonda de las Naciones Unidas sobre Comunicación para el Desarrollo. Roma. Recuperado de ftp://ftp.fao.org/docrep/fao/010/a1476s/a1476s03.pdf

Bonilla Castro, E. y Rodríguez Sehk, P. (1997). Más allá del dilema de los métodos. Bogotá: Norma-Uniandes.

Carvajal, D. (2002). Las herramientas de la artesana. Aspectos críticos en la enseñanza y aprendizaje de los CAQDAS. Qualitative Social Research, 3(2), art. 14. Recuperado de http://nbn-resolving.de/urn:nbn:de:0114-fqs0202147

Cimadevilla, G. (2005). Información ambiental, espectacularización y desconexión. En D. Mato (coord.). Políticas de economía, ambiente y sociedad en tiempos de globalización. Caracas: Universidad Central de Venezuela, Facultad de Ciencias Económicas y Sociales.

Coulon, A. (1988). La etnometodología. Madrid: Cátedra.

Del Río Sandornil, D. (1996). Elaboración de una historia de vida: complementariedad y técnicas. En E. López-Barajas (Ed.). Las historias de vida, la investigación biográfica. Madrid: Universidad Nacional de Educación a Distancia.

Food and Agriculture Organization of the United Nations (FAO) (2003). Communications and natural resource management: Experiencie and theory. Roma: Autor. 
María Victoria Rugeles Gélvez, Eliana del Rosario Herrera Huérfano, Carlos Andrés Muñoz Sandoval A la zaga de una propuesta para la combinación de metodologías de investigación

Gaitán Moya, J. A. y Piñuel Raigada, J. L. (1998). Técnicas de investigación en comunicación social. Elaboración y registros de datos. Madrid: Síntesis.

Gumucio-Dagron, A. (2004). El cuarto mosquetero: la comunicación para el cambio social. Revista Investigación y Desarrollo, 12. Recuperado de www.redalyc.org

Hammersley, A. y Atkinson, P. (2006). Etnografia: métodos de investigación. Barcelona: Paidós.

Hammersley, M. (2010). Can we re-use qualitative data via secondary analysis? Notes on some terminological and substantive Issues. Sociological Research Online, 15(1). Recuperado de http://www.socresonline.org.uk/15/1/5.html

Herrera Arango, Á. D. y Uruburu Gilède, S. (2009). Teorías, enfoques y estrategias de desarrollo: el papel de la comunicación en el cambio social. Estado del arte de la investigación en comunicación y desarrollo en Colombia, 2002-2006. Hallazgos, 12(6), 111-146. Bogotá: Universidad Santo Tomás.

Herrera Huérfano, E. (2009). Comunicación, desarrollo y medioambiente. En Memorias II Encuentro de Investigación en Comunicación. Bogotá: Uniminuto.

Leff, E. (2003). La ecología política en América Latina. Un campo en construcción. Polis, 1(5). Recuperado de http://www.redalyc.org/articulo.oa?id=30500505

Penna, J. A. y Cristeche, E. (2008). Valoración de los servicios ambientales: diferentes paradigmas. Estudios socioeconómicos de sustentabilidad de los sistemas de producción y recursos naturales. Buenos Aires: Instituto Nacional de Tecnología Agropecuaria.

Raigada Piñuel, J. L. (2002). Epistemología, metodología y técnicas del análisis de contenido. En Estudios de sociolingüistica. Madrid: Universidad Complutense de Madrid.

Rodríguez Gómez, G., Gil Flores, J. y García Jiménez, E. (2002). Metodología de la investigación cualitativa. Cuba: Prograf.

Sandoval Casilimas, C. (1996). La investigación cualitativa. Bogotá: Instituto Colombiano para el Fomento de la Educacón Superior. 
Seoánez C., M. (1997). El medioambiente en la opinión pública. Barcelona: Ediciones Mundi-Prensa.

Tezanos, A. de (1998). Una etnografía de la etnografía. Aproximaciones metodológicas para la enseñanza del enfoque cualitativo-interpretativo para la investigación social. Bogotá: Anthropos.

Valles, M. (2002). Ventajas y desafíos del uso de programas informáticos (e.g. Atlas.ti y MAXqda) en el análisis cualitativo. Una reflexión metodológica desde la grounded theory y el contexto de la investigación social española. Madrid: Universidad Complutense de Madrid.

Valles, M . (2005). Metodología y tecnologías cualitativas: actualización de un debate, desde la mirada más atenta en la obra de Barney G. Glaser. Revista Empiria, 9, 145-148.

Waisbord, S. (2001). Family tree of theories, methodologies, and strategies in the development Communications. Convergences and differences. Recuperado de http://www. comminit.com/stsilviocomm/sld-2881.html

Wenger E. (1998). Communities of practice: learning, meaning, and identity. Reino Unido: Cambridge University Press.

Wenger, E. (2004). Knowledge management as a doughnut. Recuperado de http://iveybusinessjournal.com/topics/leadership/knowledge-management-as-a-doughnut\#. Uj4L0cYz3ng

Wenger, E. (2006). Communities of practice. A brief introduction. Recuperado de http:// www.ewenger.com/theory/ 